\title{
Grain size in script and teaching: Literacy acquisition in Ge'ez and Latin
}

\author{
YONAS MESFUN ASFAHA, JEANNE KURVERS, and SJAAK KROON \\ Tilburg University
}

Received: November 6, 2007 Accepted for publication: November 15, 2008

\section{ADDRESS FOR CORRESPONDENCE}

Yonas Mesfun Asfaha, Department of Language and Culture Studies, Tilburg University, P.O. Box 90153, Tilburg 5000 LE, The Netherlands. E-mail: y.m.asfaha@uvt.nl

\begin{abstract}
The study investigated reading in four African languages that use either syllabic Ge'ez (Tigrinya and Tigre languages) or alphabetic Latin scripts (Kunama and Saho). A sample of 385 Grade 1 children were given letter knowledge, word reading, and spelling tasks to investigate differences at the script and language levels. Results showed that the syllable based Ge'ez script was easier to learn than the phoneme-based Latin despite the bigger number of basic units in Ge'ez. Moreover, the syllable based teaching of alphabetic Saho produced better results than alphabetic teaching of Kunama. These findings are discussed using the psycholinguistic grain size theory. The outcomes confirm the importance of the availability of phonological units in learning to read.
\end{abstract}

In the process of becoming literate, beginner readers across languages and orthographies primarily acquire systems of correspondences between graphic symbols and units of sound (Byrne, 1998; Share, 1995; Ziegler \& Goswami, 2006). Depending on the writing system, the representation of language units in orthography may take the phoneme, syllable or morpheme as a starting point. Although it seems evident that phonological awareness plays an important role in the development of reading in all orthographies (Ziegler \& Goswami, 2005), differences in the pace of the development of phonological recoding have been observed across languages. In a survey of early reading development in 13 European languages, Seymour, Aro, and Erskine (2003) discovered that the development of reading in opaque and inconsistent orthographies (such as English) was slower than in shallow and consistent orthographies (such as Finnish). In their seminal article on becoming literate in different languages, Ziegler and Goswami (2005) proposed the psychological grain size theory (PGST) to explain these developmental differences in reading in several European orthographies. According to the theory, differences in reading accuracy and speed observed across orthographies "reflect fundamental differences in the nature of the phonological recoding and reading strategies that are developing in response to the orthography" (Ziegler \& Goswami, 2005, p. 19).

The PGST highlights the significance of three core features in the relationship between language and orthography: the availability or accessibility of 
phonological units, the consistency of the mapping between spelling and sound, and the granularity or the grain size of the scripts. These features refer to the three core problems learners face in beginning to read. The availability problem relates to the fact that not all phonological units are equally accessible (Durgunoglu \& Oney, 1999; Gombert, 1992; Liberman, Shankweiler, Fischer, \& Carter, 1974). Consistency refers to the problem that some graphemes can have different pronunciations or some sounds different spellings. The granularity problem reflects the reality that using a bigger grain size in the orthography typically means a larger number of basic orthographic units (e.g., there are more characters in Chinese than letters in English).

In the process of acquiring early phonological recoding skills, these three problems barely operate independent of one another. For example, using a more easily accessible bigger grain size (like a syllable) in the orthography often comes with a price: the beginner reader has to learn much more units than in small unit orthographies. In addition, the basic grain size in the orthographic systems do not always coincide with the grain size of the teaching methods (Ziegler \& Goswami, 2006). Therefore, some of the important questions follow. What is the relative importance of each of the core features of availability, consistency, and granularity in beginning reading? How important is availability, for example, when compared to granularity or consistency? (For interactions of consistency and granularity in phonological dyslexia, see Wydell \& Kondo, 2003.) Ziegler and Goswami’s (2005) cross-linguistic theory emphasized the consistency of the mappings between the orthographic and phonological units to explain differences in reading development across European languages. Goswami and Ziegler (2006) also acknowledged the relative availability of smaller units (phonemes) in simple phonological structures represented with consistent orthographies (e.g., Italian or Spanish) or in languages with rich morphological structures (e.g., Turkish).

Although there was no explicit reference to the PGST, the study by Nag (2007) compared English and Kannada (an Indian alphasyllabary with 470 Akshara symbols) beginning reading skills. The outcomes of the study pointed to the impact of granularity as the study showed that learning to read in Kannada was slowed down by the large number of Akshara syllable symbols the children had to learn. Winskel and Widjaja (2007) studied the grain size adopted by beginner readers and spellers of Indonesian, a language where the syllable is salient and the alphabetic orthography transparent. They found out that reading and spelling were primarily acquired at the phoneme level but were augmented by acquisition of reading and spelling at the syllable level as well.

Despite the availability of cross-linguistic studies (Lee, Uttal \& Chen, 1995; Nag, 2007; Seymour et al., 2003), the application of the PGST in learning nonalphabetic orthographies has been very rare. Studies across different scripts and languages are often complicated by the fact that comparisons are mainly possible in different cultural and educational traditions. There are comparative investigations of early reading acquisition in different languages taught within a national curriculum (see Bruck, Genesee, \& Caravolas, 1997; Ellis \& Hooper, 2001), although these studies deal with alphabetic languages only (English, French, and Welsh).

The current study especially investigated the relative importance of two of the core features of the PGST (i.e., availability and granularity) to early reading in different languages and scripts in Eritrea, a multilingual African country. The 
study compared early reading and spelling skills in four languages that employ two different systems of writing: the alphasyllabic Ge'ez or Ethiopic and the alphabetic Latin scripts.

Because PGST emphasizes the phonological structures and the consistency with which those structures are coded in the orthography (Ziegler \& Goswami, 2005), we briefly describe the languages in the study, their orthographies and the teaching methods used before presenting the empirical study and the results of the comparisons.

\section{GE'EZ AND LATIN ORTHOGRAPHIES}

Our study compared beginning reading and spelling in four different languages in Eritrea, a country in the Horn of Africa, where the educational policy allows the use of the country's nine languages and three scripts (Ge'ez, Latin alphabet, and Arabic) in primary education. Four of these languages (Saho, Kunama, Tigrinya, and Tigre) are the focus of this study. Saho and Kunama are written in the Latin alphabetical script, and Tigrinya and Tigre in the alphasyllabic Ge'ez script.

The four languages in this study share a simple phonological structure. The Semitic languages Tigrinya and Tigre allow vowel (V), consonant-V (CV), and CVC combinations as syllables, with clusters of consonants broken up with the insertion of a vowel to conform to the CV and CVC syllable structure (Raz, 1983). The Tigre language occasionally has the CCV or more precisely the tCV syllabic structure, for example, tgasa (he sat down; S. Idris, personal communication, July 2007). The Cushitic Saho also has a simple syllabic structure and does not allow consonant clusters (Banti \& Vergari, 2006). Typical Saho contains V, CV, VC, and CVC syllables (e.g., alsa, moon, faras, horse). Nilo-Saharan Kunama has an even simpler CV open syllable structure, especially with nouns. Thus, a loanword like kubbayat (cup) from Tigre, for example, adds a vowel at the end, kubbayata, to become a Kunama noun (Abraha, 2005). All the languages have rich morphology.

Although a systematic analysis is difficult to find, the orthographies of the four languages show regularity in the way orthographic units represent either phonemes (Saho and Kunama) or syllables (Tigrinya and Tigre). The Ge'ez orthographies of Tigrinya and Tigre also use 'single consonant' symbols (with silent vowels) to represent syllable endings in the CVC-syllables.

Tigrinya and Tigre orthographies are based on the script of Ge'ez, an alphasyllabary originally used to write a Semitic language by the same name that is now limited to Orthodox Church liturgical use. A single Ge'ez letter, commonly called fidel, consists of a core consonant and a vowel. The seven vowels form the columns of a traditional ordering of consonants in rows with vowel changes indicated by a slight change of the diacritic to consonant entry in each row. The resulting table consists of many syllable symbols (245 in Tigrinya, 205 in Tigre) in a predictable pattern (see Figure 1 for sample fidel symbols). The Tigrinya and Tigre orthographies do not represent gemination of consonants. Tigrinya has 35 consonants and seven vowels, where some of the Tigrinya consonants are labialized variants of the basic Ge'ez consonants. Tigre has 25 consonants and seven vowels. The Tigre orthography recognizes the vowels in the first and fourth column of the matrix as the same vowel with different lengths. Unlike other Semitic orthographies such as Hebrew, the Ge'ez orthographies of Tigre and Tigrinya are transparent in how 
Asfaha et al.: Grain size in script and teaching

\begin{tabular}{|c|c|c|c|c|c|}
\hline $\begin{array}{l}\boldsymbol{U} \\
\text { ha }\end{array}$ & $\begin{array}{l}\boldsymbol{v} \\
\text { hu: }\end{array}$ & $\begin{array}{l}\text { Y. } \\
\text { hi: }\end{array}$ & $\begin{array}{l}y \\
\text { ha: }\end{array}$ & $\begin{array}{l}\boldsymbol{y} \\
\text { he: }\end{array}$ & $\begin{array}{l}y \\
h(2)\end{array}$ \\
\hline $\begin{array}{l}n \\
\text { la }\end{array}$ & $\begin{array}{l}\boldsymbol{A} . \\
\text { lu: }\end{array}$ & $\boldsymbol{\Lambda}_{\mathrm{li}}$ & $\begin{array}{l}\lambda \\
\text { la: }\end{array}$ & $\begin{array}{l}\boldsymbol{\lambda} \\
\text { le: }\end{array}$ & $\begin{array}{l}a \\
I(0)\end{array}$ \\
\hline OD & oo. & $\sigma q$. & $\sigma q$ & $\sigma g$ & $90^{\circ}$ \\
\hline $\mathrm{ma}$ & $\mathrm{mu}$ : & $\mathrm{mi}:$ & ma: & me: & $m(0)$ \\
\hline $\begin{array}{l}+ \\
\text { ta }\end{array}$ & $\begin{array}{l}\text { H: } \\
\text { tu: }\end{array}$ & $\begin{array}{l}\mathbf{t} \\
\text { ti: }\end{array}$ & $\begin{array}{l}f \\
\text { ta: }\end{array}$ & $\begin{array}{l}\text { ts } \\
\text { te: }\end{array}$ & $\begin{array}{l}7 \\
t(a)\end{array}$ \\
\hline
\end{tabular}

Figure 1. A sample of Ge'ez fidel symbols from the Tigrinya table.

they represent CV fidel symbols, although the vowel diacritics are not always graphically put in a predictable linear pattern (e.g., see second row, Figure 1).

Latin alphabetic Saho orthography consists of 31 consonant letters (out of which nine are digraphs) and five vowel letters. Long vowels and consonant geminations in Saho are represented by doubling of the letters. In the Latin alphabetic Kunama orthography, there are 20 consonant and five vowel letters with length of vowel and consonant gemination indicated by doubling of the letters. There are two digraphs in the orthography. Starting from 2004, the Kunama school orthography has incorporated two diacritics to represent a high and a rising tone (J. Abraha, personal communication, July 2007). Until now, the tone diacritics have only been used in the lower grades of primary school.

\section{Teaching methods in Ge'ez and Latin}

Ziegler and Goswami (2006), like many others, warned against potential problems arising from sociocultural differences in conducting cross-linguistic research (see also Lee et al., 1995). Such differences include variations in educational traditions, teacher qualifications, teaching methods, and the availability of resources. Eritrea is an excellent context for cross-linguistic and cross-scriptal research because all the schools employ the same national curriculum, comparable instructional materials, and similar teaching methods. Teacher qualifications are comparable (1 year of teacher training) and group sizes are usually large over the entire country (on average 40-60 children in a classroom).

Textbooks are prepared in each of the nine languages by language panels (groups of language and education experts) following the same scope and sequence descriptions that are based on the national curriculum. Each textbook is accompanied by a teacher guide. The Grade 1 textbooks for the students in all the four languages of the study are similar in content and structure. The Grade 1 textbooks start with the introduction of a vowel in the case of Latin alphabet or a CV fidel symbol in the case of Ge'ez, appearing together with a picture of an object or animal the name of which begins with the same letter or fidel symbol. The rest of the letters 
and fidel symbols are gradually presented in the same manner over the pages of the whole primer. Teaching of the Tigrinya and Tigre letters to beginner readers emphasizes familiarization with the fidel table, which has a demanding number of characters, although not as many as the Akshara syllable symbols in Kannada (Nag, 2007). In all textbooks (Ge'ez and Latin), new graphemes or fidel symbols are introduced together with a word and a picture, and followed by exercises in repetition and blending of graphemes or fidel symbols thus far presented.

Teaching the alphabetic Latin and the alphasyllabic Ge'ez has always been influenced by the traditional 'chanting after the teacher' (Wright, 2001), with its roots in religious Ge'ez and Arabic schools and its suitability to large class sizes. The current classroom practice revolves around drill-oriented learning and memorization of letters and syllable symbols. This appears to be leaning towards a skills approach. In both types of classrooms, syllable blending, simple word decoding, and gradual introduction of short sentences are part of the instruction.

Although the content of the primers in Kunama and Saho is comparable, the introduction and teaching of the graphemes differs slightly. Teaching of the alphabetic Saho letters is centered on syllables. For example, the introduction of the letter " $\mathrm{k}$ " immediately starts with the syllable $\mathrm{ka}$. The reading instruction goes on to name the rest of the CV structures combining the consonants with the five vowels $(k a, k u, k i, k e, k o)$. The exercises that follow the introduction of the letters are syllable blending $(k o+b e=k o b e ; l a a+m a=l a a m a)$. The next consonant is introduced in the same way $(l a, l u, l i, l e, l o)$. Kunama orthography teaching is more comparable to what is practiced in the teaching of the Latin alphabet, that is, there is an effort to achieve one to one phoneme-letter correspondences. Exercises on phoneme and syllable blending are commonly used to highlight length (vowel and consonant) and tone variations.

The current study compared the spelling and reading results of children from these four languages that use two scripts with different grain sizes, the syllable, and the phoneme. The study aimed (a) to compare reading and writing skills of Grade 1 children in the alphasyllabic Ge'ez orthographies with the alphabetic ones and (b) to compare, within the alphabetic orthographies, the reading and writing skills of Grade 1 children in the two languages that differed in the grain size emphasized in the initial teaching. Some Grade 4 comparisons are also added to aid interpretation of the Grade 1 outcomes. The Grade 4 Kunama results were in the old Kunama orthography that lacked tone diacritics.

As there have been studies that suggested that syllables are much more accessible than phonemes (Bertelson, 1986; Durgunoglu \& Oney, 1999; Gombert, 1992; Liberman et al., 1974; Morais, Cary, Alegria, \& Bertelson, 1979), we expect that children learning to read the syllable-based Ge'ez orthographies to do better in word reading and spelling tasks than children in the phoneme-based Latin orthographies. From the availability point of view, this leads to the prediction that Ge'ez script students will have higher reading and spelling scores than Latin alphabet students. However, there are 205 or 245 symbols in the Tigre and Tigrinya Ge'ez orthographies compared to the 28 or 34 letters in the Kunama and Saho Latin orthographies. In terms of the big number of syllable symbols, the Ge'ez script may prove to be a disadvantage to the beginner reader (compare Nag, 2007). From this granularity point of view, we expect Ge'ez fidel symbols knowledge 
to be lower than the Latin letter knowledge and we expect reading and spelling results in Ge'ez to be affected accordingly.

Regarding the consistent Latin orthographies of Kunama and Saho, we expect that the Saho syllable teaching method will be more advantageous to beginner readers than the Kunama phoneme-based teaching method.

In summary, the study aimed to investigate the impact of the grain size at two levels. First, it looked at the grain size of the script in relation to the number of symbols by comparing the alphasyllabic Ge'ez with the Latin alphabet. Second, the effect of the grain size of initial teaching in Saho and Kunama alphabetic orthographies was compared.

\section{METHODS}

\section{Participants}

The study included 385 Grade 1 children randomly selected from 29 Kunama, Saho, Tigre, and Tigrinya medium schools. In some cases, more than one classroom was sampled from a single school. Children whose mother tongue differed from the language of instruction in the schools were excluded from the study. To minimize teacher effects, we randomly selected 10-12 children from each of the classrooms. Because Walter and Davis (2005) revealed very poor reading performance among primary school children in Eritrea, we used a stratified sampling strategy to circumvent floor effects. About 4 to 5 children of the "high achieving" (ranking in the upper ten in overall classroom achievement) and slightly more students (6 to 7) from the rest of the class were randomly selected. Therefore, about $40 \%$ of the children in the sample came from the 10 best children in each of the classrooms, and the remaining from the rest of the students in the classrooms. From the same schools, 206 Grade 4 students were assessed using the same word reading test as in Grade 1 (see Table 1). The schools were selected from urban and rural geographical areas where the four languages are dominant. The physical conditions of the rooms, the availability of teaching materials, and the number and the professional training of teachers were comparable across the languages. For example, a minimum of $81 \%$ (Kunama teachers) and a maximum of $98 \%$ (Tigre) of the teachers in the schools under study had received year-long teacher training before they started teaching.

\section{Instruments}

Grapheme or fidel symbol knowledge, word reading, and spelling were the main instruments developed for use in this research. All instruments were piloted in all the languages of the study in different locations.

Background questionnaire. This consisted of questions on the child's personal data (first language, age, classroom repetition, ranking, grade, sex, and preschool experience), parents' education (years of schooling) and social (job) background. 
Asfaha et al.: Grain size in script and teaching

Table 1. Number of schools and students for each of the languages

\begin{tabular}{|c|c|c|c|c|c|c|}
\hline \multirow[b]{2}{*}{ Scripts } & \multirow[b]{2}{*}{ Languages } & \multirow[b]{2}{*}{ Schools } & \multicolumn{2}{|c|}{ Grade 1} & \multicolumn{2}{|c|}{ Grade 4} \\
\hline & & & $N$ & $\%$ & $N$ & $\%$ \\
\hline \multirow[t]{2}{*}{ Alphabetic } & Kunama & 5 & 102 & 26.5 & 59 & 28.6 \\
\hline & Saho & 7 & 87 & 22.6 & 49 & 23.8 \\
\hline \multirow{3}{*}{ Syllabic } & Tigre & 6 & 86 & 22.3 & 49 & 23.8 \\
\hline & Tigrinya & 11 & 110 & 28.6 & 49 & 23.8 \\
\hline & Total & 29 & 385 & 100.0 & 206 & 100.0 \\
\hline
\end{tabular}

Letter knowledge. Because knowledge of graphemes and syllable symbols (Lee et al., 1995; Nag, 2007; Seymour et al., 2003) was found to be a predictor of progress in learning to read in alphabetic and alphasyllabic orthographies, a list of 25 randomly selected letters and symbols from each of the four languages formed the letter and syllable symbols knowledge tests. In the Latin orthography tests, the letters were presented in clear lower case. For the Ge'ez script tests, a list of 25 fidel symbols was prepared (Ge'ez does not differentiate between upper case and lower case). Two practice items preceded the test items. The students were asked to name the letters. Both the names and sounds of the letters were considered correct.

Word reading. Many scholars (Hambleton, 2005; Lee et al., 1995) acknowledged the difficulty of constructing comparative tests in different languages and different scripts, especially when beginning reading and spelling was the focus. Comparative test construction, therefore, has to be based on careful analysis of the curriculum of each language or script. In a single national curriculum in Eritrea, textbook analysis was a straightforward and valid way of proceeding with test construction, as schools use comparable textbooks for beginning reading in each of the languages. In preparing the reading tests, all words in the textbooks for Grades 1-5 were listed for each of the languages. These lists of words constituted, for each language, a computerized dictionary (Lee et al., 1995) from which the word reading and spelling tasks were prepared.

Every word from the Grade 1 textbook was entered into the computerized dictionary. Subsequently, every new word from the textbook of the next grade level was added to the dictionary together with a note indicating the grade level. Considering the early readers' perspective and the rich morphology of the languages involved, inflections of root words (e.g., Wedi Tigrinya for "boy" and Awedat "boys") were mostly counted as new entries. The 200 word items in the word reading tests for each of the four languages were then randomly selected from these dictionaries. Out of the 200 words in the word reading task, 120 were selected from the Grade 1 part of the list and the rest from higher grade-level entries in the dictionary. This was an attempt to include words that children have been exposed to in their first year at school but also words that they are likely to encounter in subsequent school years. The first 70 words in the word list were placed in a single page in 
Asfaha et al.: Grain size in script and teaching

Table 2. Reliability (Cronbach alpha) of the instruments in each language

\begin{tabular}{|c|c|c|c|c|c|}
\hline & $\begin{array}{c}\text { Kunama } \\
(N=102)\end{array}$ & $\begin{array}{c}\text { Saho } \\
(N=87)\end{array}$ & $\begin{array}{c}\text { Tigre } \\
(N=86)\end{array}$ & $\begin{array}{c}\text { Tigrinya } \\
(N=110)\end{array}$ & $\begin{array}{c}\text { Overall } \\
(N=385)\end{array}$ \\
\hline Letter knowledge & .95 & .95 & .89 & .94 & .93 \\
\hline \multicolumn{6}{|l|}{ Word reading } \\
\hline Grade 1 & .99 & .99 & .98 & .99 & .99 \\
\hline Grade 4 & .96 & .98 & .98 & .90 & .98 \\
\hline Spelling & .97 & .93 & .92 & .94 & .95 \\
\hline
\end{tabular}

two columns and the remaining words in the next page to avoid possible feelings of panic with those students poorly progressing through the list. The same word reading test was used in Grade 4.

Spelling. The 20 items for the spelling test in each of the languages were randomly selected from the 200 items list in the word reading test. Half of the 20 spelling items were from the Grade 1 part of the word reading test and the rest were from the upper grades.

The internal consistency of all the tests was good in general and in each of the languages (see Table 2).

\section{Procedure}

The tests were administered with the help of multilingual research assistants and multilingual teachers from the schools that participated in the study. Both groups of assistants were given training ahead of the testing and were closely monitored by the principal researcher on all sites throughout the recording period. Only speakers with one of the four languages (Kunama, Saho, Tigre, and Tigrinya) as their mother tongue were accepted as test takers. All the tests were administered with individual students in or around their classrooms using the respective languages of the children as media of communication. The child was handed the test copy and allowed to practice on the practice items before the examiner urged the first grader to move to the test items. In the grapheme or fidel symbol knowledge test, the examinee mentioned the letters or symbols as the research assistant recorded the answers in the examiner's copy. The word reading test was conducted in a similar way except that the child was reminded to read as far down the list of words as possible in the 3 min provided for the timed word reading task. The same procedures were used in the same word reading test given to Grade 4 children.

Words in the spelling test to Grade 1 were read to children who then attempted the spelling on the answer sheet while the examiner recorded the results. Dictating letters, words, and sentences to students is still widely practiced in the schools, which meant in practice there was little or no need to put single words said by the examiner in context in most parts of the spelling test. 
Asfaha et al.: Grain size in script and teaching

Table 3. Background of Grade 1 participants and parents' mean years (standard deviations) of education

\begin{tabular}{lccccccc}
\hline \hline & $\begin{array}{c}\text { Tigrinya } \\
(N=110)\end{array}$ & $\begin{array}{c}\text { Tigre } \\
(N=86)\end{array}$ & $\begin{array}{c}\text { Ge'ez } \\
\text { Total }\end{array}$ & $\begin{array}{c}\text { Kunama } \\
(N=102)\end{array}$ & $\begin{array}{c}\text { Saho } \\
(N=87)\end{array}$ & $\begin{array}{c}\text { Latin } \\
\text { Total }\end{array}$ & $\begin{array}{c}\text { Overall } \\
\text { Total }\end{array}$ \\
\hline Male $(\%)$ & 54.5 & 61.6 & 57.7 & 48.0 & 78.2 & 61.9 & 59.7 \\
Female $(\%)$ & 45.5 & 38.4 & 42.3 & 52.0 & 21.8 & 38.1 & 40.3 \\
$\begin{array}{l}\text { Preschool } \\
\quad(\%)\end{array}$ & 78.2 & 88.4 & 82.7 & 37.3 & 82.8 & 58.2 & 70.6 \\
Mean age & 6.64 & 7.68 & 7.11 & 7.92 & 8.09 & 8.0 & 7.56 \\
& $(0.76)$ & $(0.76)$ & $(0.92)$ & $(1.04)$ & $(1.85)$ & $(1.47)$ & $(1.30)$ \\
Education & & & & & & & \\
$\quad$ Father & 2.68 & 1.97 & 2.37 & 2.58 & 1.27 & 1.97 & 2.18 \\
& $(4.04)$ & $(3.29)$ & $(3.74)$ & $(4.68)$ & $(2.49)$ & $(3.89)$ & $(3.80)$ \\
\multicolumn{1}{c}{ Mother } & 3.08 & 1.90 & 2.58 & 1.54 & 1.01 & 1.28 & 1.95 \\
& $(4.23)$ & $(2.48)$ & $(3.63)$ & $(2.98)$ & $(2.08)$ & $(2.61)$ & $(3.24)$ \\
\hline \hline
\end{tabular}

\section{RESULTS}

We first examine the background data of the Grade 1 sample of the four language groups (see Table 3 ) before proceeding to comparisons of grain sizes in the scripts and the teachings.

The two script groups (Ge'ez and Latin) did not differ significantly in the number of boys and girls in the groups, $\chi^{2}(1, N=358)=.72, n s$. They did differ, however, in the percentages of children that had preschooling, $\chi^{2}(1, N=$ $358)=27.74, p<.001$. The two script groups also differed significantly in age, $t$ $(314.291)=-7.03, p<.001$, and in mother's years of schooling, $t(341.694)=$ $-3.95, p<.001 .^{1}$ There was no significant difference between the script groups in the educational background of the fathers. The Tigrinya were mainly Orthodox Christians, whereas the Tigre and Saho were predominantly Muslims. The Kunama were partly Christians (Protestant or Roman Catholic) and partly Muslims. Preschool attendance for the Tigrinya referred mainly to Orthodox Church recitation-based religious schools and to kindergartens in urban areas; for the Tigre, Saho and Muslim Kunama it mainly meant recitation based Qur'anic schools.

\section{Grain size in scripts}

To investigate beginning reading skills in the two scripts that differed in the grain size of the basic unit (syllable vs. phoneme), we compared results of letter knowledge, word reading, and spelling tasks across Latin (Kunama and Saho, $N=$ 189) and Ge'ez (Tigre and Tigrinya, $N=196$ ). The Cohen $d$ value was calculated to measure effect sizes. The two script groups differed significantly in preschool experience of the children, in age and in educational level of the mothers (see Table 3). Table 4, therefore, presents the results of comparisons and outcomes of the analysis of variance, with age, preschooling and mother's educational level as covariates. $^{2}$ 
Asfaha et al.: Grain size in script and teaching

Table 4. Grade 1 script group mean results (standard deviations), F values, and Cohen $d$

\begin{tabular}{|c|c|c|c|c|c|c|}
\hline & Range & $\begin{array}{c}\text { Total } \\
(N=358)\end{array}$ & $\begin{array}{l}\text { Alphabetic } \\
(N=178)\end{array}$ & $\begin{array}{c}\text { Syllabic } \\
(N=180)\end{array}$ & $F(1,354)$ & $d$ \\
\hline Letter knowledge & $0-25$ & $\begin{array}{l}17.53 \\
(6.84)\end{array}$ & $\begin{array}{l}18.61 \\
(6.66)\end{array}$ & $\begin{array}{l}16.45 \\
(6.86)\end{array}$ & $9.11 * *$ & 0.33 \\
\hline Spelling & $0-20$ & $\begin{array}{c}7.38 \\
(6.82)\end{array}$ & $\begin{array}{l}5.49 \\
(6.51)\end{array}$ & $\begin{array}{c}9.24 \\
(6.62)\end{array}$ & $21.27 * * *$ & 0.57 \\
\hline Word reading & $0-115$ & $\begin{array}{c}31.19 \\
(31.16)\end{array}$ & $\begin{array}{c}22.72 \\
(29.12)\end{array}$ & $\begin{array}{c}39.57 \\
(30.93)\end{array}$ & $18.09 * * *$ & 0.55 \\
\hline
\end{tabular}

$* * p<.01 . * * * p<.001$.

The results revealed that the mean scores of Ge'ez orthographies were significantly higher than Latin ones in the spelling and word reading tasks $(p<.001)$. Notwithstanding the overall low reading rate (on average, 31 words $/ 3 \mathrm{~min}$ ), the difference between Ge'ez (about 40 words/3 min) and Latin ( 23 words) was about 17 words, with medium to large effect sizes $(d=0.57$ and 0.55$)$. The scores on letter knowledge, however, were significantly higher in Latin than in Ge'ez. On average, $70 \%$ of the 25 graphemes or fidel symbols in the task were read correctly. The average Latin score was significantly higher than the Ge'ez score, with a medium effect size $(d=0.33)$.

Although the longer list of fidel symbols in the Ge'ez script initially put children at a disadvantage, the effect of the easy to access and blend larger grain size (syllable) was nevertheless reflected in higher reading and spelling results in Ge'ez. Children appeared to be learning to read and spell faster in Ge'ez than in Latin scripts. As was evident in the comparisons of the consistent with the less consistent European orthographies (Ziegler \& Goswami, 2005), the syllable availability advantage might fade away as children progress in reading. Taking this into consideration, the analysis next compared the Ge'ez and Latin results in Grade 4 by first investigating the groups' background variables of age, preschooling, and educational levels of the parents. In all cases, the groups differed significantly. The Grade 4 Ge'ez script students were younger than the Latin script students, $t(202.676)=-5.24, p<.001$, significantly more Ge'ez script students had preschooling, $\chi^{2}(1, N=171)=15.94, p<.001$, and both parents (fathers and mothers) of the Ge'ez script students had on average more years of education, respectively, $t(169.618)=2.81, p<.01$ and $t(160.184)=3.79, p<$ .001 . Therefore, age, preschooling, and parental educational levels were included as covariates. ${ }^{3}$ Table 5 presents the outcomes of comparisons of the two script groups.

As expected, the differences between Ge'ez and Latin script groups in Grade 4 were not significant. The two script groups scored nearly the same. The advantages of the syllabic Ge'ez script over the alphabetic Latin that were observed in the Grade 1 results seemed to diminish when learning progressed. 
Table 5. Grade 4 script group mean results (standard deviations), $F$ value, and Cohen $d$

\begin{tabular}{lcccc}
\hline \hline & $\begin{array}{c}\text { Syllabic } \\
(N=85)\end{array}$ & $\begin{array}{c}\text { Alphabetic } \\
(N=86)\end{array}$ & $F(1,165)$ & $d$ \\
\hline Word reading & $\begin{array}{c}115.49 \\
(36.54)\end{array}$ & $\begin{array}{c}118.48 \\
(30.87)\end{array}$ & 2.21 & 0.08 \\
\hline \hline
\end{tabular}

Table 6. Grade 1 Kunama and Saho mean results (standard deviations), $F$ values, and Cohen $d$

\begin{tabular}{lcccc}
\hline \hline & $\begin{array}{c}\text { Kunama } \\
(N=86)\end{array}$ & $\begin{array}{c}\text { Saho } \\
(N=75)\end{array}$ & $F(1,157)$ & $d$ \\
\hline Letter knowledge & 17.03 & 20.00 & $4.36^{*}$ & 0.46 \\
Spelling & $(7.24)$ & $(5.88)$ & & \\
& 2.55 & 8.55 & $38.93^{* * *}$ & 1.03 \\
Word reading & $(5.01)$ & $(6.26)$ & & \\
& 8.15 & 38.44 & $52.26^{* * *}$ & 1.95 \\
& $(16.97)$ & $(31.12)$ & & \\
\hline \hline
\end{tabular}

$* p<.05 . * * * p<.001$.

\section{Grain size in teaching}

Kunama and Saho share a consistent Latin script and a simple syllabic language structure. The two languages differ, however, in the grain size used in the initial teaching. The Saho teaching method emphasized the syllable while Kunama teaching focused on the phoneme. To see the effect of the grain size in teaching, we compared the Saho and Kunama results. Because the groups differed significantly in preschool experience (significantly more Saho children had preschooling), $\chi^{2}(1, N=161)=39.96, p<.001$, and educational levels of the fathers, $t(133.199)=2.26, p=0.03$, covariance analysis was used with language as independent variable and preschool experience and father's educational level as covariates. $^{4}$

The results of the comparisons of the two Latin orthographies are presented in Table 6. The results revealed that Saho children scored significantly higher than Kunama children did in all tasks ( $p<0.05$ for grapheme knowledge, and $p<$ 0.001 for spelling and word reading). The effect sizes are high for word reading and spelling, and medium for letter knowledge.

The results indicated that it made sense (with languages of simple syllabic structure) to use the syllable as a basis for teaching beginning reading even with alphabetic orthographies. The higher Saho results revealed that keeping the traditional syllabic way of teaching was comparatively effective at the beginning stages of reading.

To see whether the syllable teaching advantage also occurs at a later stage, word reading results from Grade 4 (where the Kunama word reading test was 
Asfaha et al.: Grain size in script and teaching

Table 7. Grade 4 Kunama and Saho mean results (standard deviations), $F$ values, and Cohen d

\begin{tabular}{lcccc}
\hline \hline & $\begin{array}{c}\text { Kunama } \\
(N=43)\end{array}$ & $\begin{array}{c}\text { Saho } \\
(N=43)\end{array}$ & $F(1,80)$ & $d$ \\
\hline Word reading & $\begin{array}{l}113.30 \\
(26.66)\end{array}$ & $\begin{array}{c}123.65 \\
(34.01)\end{array}$ & 1.43 & 0.08 \\
\hline \hline
\end{tabular}

a reproduction of the Grade 1 list without tone diacritics) in Kunama and Saho were compared. (Although the addition of tone diacritics in Kunama Grade 1 has made the orthography much more consistent, the cognitive load [memorizing] for the children became consequently higher, because the number of vowel representations, for example, increased threefold when compared to the old system. What these new additions mean to performances in Grade 1 Kunama need further investigation.)

Table 7 presents the Grade 4 Kunama and Saho results, controlling again for significant differences in background variables of age, $t(106)=5.61, p<.001$, preschooling, $\chi^{2}(1, N=86)=53.08, p<.001$, and parental (fathers and mothers) educational levels, respectively, $t(60.014)=2.46, p=.02$ and $t(84.832)=2.55$, $p=0.01 .^{5}$

Although the average word reading scores of the Grade 4 Saho readers were higher than the Kunama results, the differences were not significant.

\section{DISCUSSION}

According to the PGST, the three core problems beginner readers have to face are availability (how easily accessible are phonological units), consistency (how much deviations are there from the one to one phoneme-graphemes correspondence), and granularity (how big is the inventory, because in systems based on bigger grain sizes the beginner reader has to learn more units). Using these features of the PGST, the research set out to explain differences in results in early reading in four simple structured languages in Eritrea. The languages used the alphasyllabic Ge'ez and the alphabetic Latin scripts. Although the literacy instructions in these languages were provided within one national curriculum, there were slight variations in how letters within the alphabetic scripts were introduced to readers.

\section{Alphasyllabic and alphabetic scripts}

The results indicate that children taught to read and write in the Ge'ez syllablebased script show significantly better results in word reading and spelling than children trained in the phoneme-based Latin orthographies. Although Ziegler and Goswami (2005) warned against the simple "large unit is better than small unit" comparisons, the results here reveal that the Ge'ez large unit-based script is easier to acquire than the phoneme level system of Latin. 
Syllabaries are known for their extensive inventory of syllable symbols, in the case of Ge'ez, the fidel symbols. It was the "extensive orthography" of Kannada compared to "contained" English (Nag, 2007) that dragged the development of reading in Kannada children. In this study, the "extensive" list of fidel symbols does not appear to be a disadvantage when compared with the "contained" Latin orthographies. Although letter knowledge is better in Latin orthographies, that knowledge does not easily convert into higher scores for word reading or spelling in the Latin orthographies. These results point toward the overarching theme of accessibility of the syllable. This advantage in availability appears to be there for beginner readers in Ge'ez where there is a systematic way of adding vowel diacritics on basic consonant symbols. As blending syllables is easier than blending phonemes, knowledge of fidel symbols, however low, translates into better word reading and spelling in Ge'ez than higher knowledge of letters does in the Latin orthographies. The syllable-based orthographies are compensated for the disadvantage of the big inventory of fidel symbols with the ease of accessibility of the basic writing unit of the system. Nevertheless, some other features of Ge'ez deserve further investigation. For instance, what the existence of "single consonants" (CV symbols with weak realization of the vowels at syllable ends) in the fidel symbols system means to awareness of subsyllable units and how this may impact early reading need further study. Further study is also needed to investigate the effect of the simple syllable structure and the consistency of the syllabic structure on small unit awareness.

The outcomes reveal that the advantages of large grain size reading mainly affect the beginning stages of learning to read and spell. The initial differences in reading performances between the large unit and small unit writing systems do no longer exist at Grade 4.

\section{Syllablic and alphabetic teaching}

The importance of availability is also reflected in the significant differences between Saho and Kunama reading scores. Although both languages (with simple syllabic structures) use the phoneme-based Latin script, Saho teaching, at least at the initial stage, was based on the consonant-vowel combination teaching style, whereas Kunama stressed the phoneme-letter correspondences. The outcomes show that this puts Saho children in an advantageous position. Although successful phonological recoding requires a shared grain size in the orthography and phonology (Goswami, Ziegler, Dalton, \& Schneider, 2003), the alphabetic small unit orthography in Saho is mediated by a large unit teaching that replaces phoneme-letter by syllable-letter correspondences.

This big unit teaching of small unit orthography seems to contribute to better word reading and spelling skills among Saho beginner readers. Unlike Saho teaching, the Kunama instruction emphasizes phoneme-letter correspondences. The lower scores across the different tasks in Kunama, therefore, are indications of the unavailability of the phoneme at the teaching level. In Grade 4, the Kunama and Saho results are comparable, indicating at the possible loss of the initial Grade 1 Saho syllable teaching advantage as readers progress in their learning. However, the Grade 1 Kunama results could have also been affected by the implementation 
of the new tone orthography in Kunama schools, for example, teachers' lack of familiarity with the tone diacritics. As mentioned earlier, further investigation is needed.

Another point regarding Kunama that also requires further investigation is whether the small unit orthography and teaching contributes to better reading abilities at a later stage of the children's primary education (after 5 years of literacy instruction). As it has been established that phoneme level training (Ziegler \& Goswami, 2006) benefits learners from all languages, even readers of the most complex orthographies such as English, it is possible that the small unit instruction in Kunama at the beginning might be beneficial at a later stage.

\section{Implications}

The findings show higher results of reading and spelling in the syllable-based Ge'ez orthographies, although the number of basic units to learn is much higher in Ge'ez than in Latin. It is therefore possible to argue that this is the result of the ease of accessibility of larger units. Among the core problems of the PGST considered by the study, these results suggest availability counted more in influence than granularity in the initial stages of beginning to read and spell. As long as the inventory of signs is systematic and reasonably sized in number (compare Nag, 2007), the easy to access and blend syllables compensate for the price of larger inventory of basic orthographic units that children have to learn.

When the salient feature (i.e., syllable) of the language is represented not in the orthography but in the large unit teaching, as in the case of Saho, then the large size in teaching (which the simple structure of the language allows) leads to better decoding of words. This slightly varies from the recommendations of the PGST, where in teaching consistent orthographies, the theory predicts that the use of small grain size teaching works better than teaching based on large grain size (Ziegler \& Goswami, 2005).

This availability advantage holds true for both a writing system that is basically syllabic as for a phoneme-based Latin alphabet that adopts the accessible syllable in teaching, ignoring more or less the phonemic basis of the script.

The syllable teaching method may only be applied, however, when the simple syllabic structure of the language allows it. For example, it may not work for English, as the latter has complex syllabic structure (with highly inconsistent orthography) that may not always allow teaching of CV units. On the other hand, the simple structures and consistent orthographies in Spanish and Italian may permit syllable teaching of the alphabetic scripts. This practice of syllablebased teaching is already known in the traditional CV-style recitations of Spanish alphabets (Pollard-Durodola et al., 2004).

If the latter outcome could be confirmed by more research, it may have implications for literacy instructions in simple syllabic structure languages in Africa and in the rest of the world that have adopted the Latin alphabetic script. The suggestion might be to teach reading and writing in the Latin alphabet using the easy to access and blend syllable as a starting point, a suggestion that has been put forward earlier as well (Gleitman \& Rozin, 1977). 
Asfaha et al.: Grain size in script and teaching

\section{ACKNOWLEDGMENTS}

This project was supported by Grant W-39-307 from the Netherlands Foundation for the Advancement of Tropical Research (NWO-WOTRO).

\section{NOTES}

1. Because Levene's test indicated that the variances were not homogeneous, we present the appropriate $t$ values and degrees of freedom.

2. There is no significant main effect of the covariates age and mother's educational background on the scores, but there is an effect of preschool experience on the word reading score, $F(1,165)=6.73, p<.01$, but not on grapheme knowledge and spelling.

3. Of the covariates, only the factor age revealed a significant main effect on reading scores, $F(1,165)=6.15, p<.01$; preschool and parental educational level did not ( $p=$ $.18, .33$, and .45 for preschool experience, father's educational level, and mother's educational level, respectively).

4. There were no significant main effects of the covariates preschool experience ( $p=$ $.34, .41$, and .26 for grapheme knowledge, spelling, and word reading, respectively) and father's educational level ( $p=.61, .65$, and .94 , respectively).

5. There was no significant main effect of age $(p=.14)$, preschool experience $(p=.59)$, and parental educational level ( $p=.78$ and .17 for father's and mother's educational levels, respectively).

\section{REFERENCES}

Abraha, J. (2005). Kunama dialects and morphology. Journal of Eritrean Studies, 4, $28-44$.

Banti, G., \& Vergari, M. (2005). A sketch of Saho grammar. Journal of Eritrean Studies, 4, 100-131. Bertelson, P. (1986). The onset of literacy: Liminal remarks. Cognition, 24, 1-30.

Bruck, M., Genesee, F., \& Caravolas, M. (1997). A cross-linguistic study of early literacy acquisition. In B. A. Blachman (Ed.), Foundations of reading acquisition and dyslexia. Mahwah, NJ: Erlbaum.

Byrne, B. (1998). The foundation of literacy. The child's acquisition of the alphabetic principle. Hove: Psychology Press.

Durgunoglu, A. Y., \& Oney, B. (1999). A cross-linguistic comparison of phonological awareness and word recognition. Reading and Writing, 11, 281-299.

Ellis, N. C., \& Hooper, A. M. (2001). Why learning to read is easier in Welsh than in English: Orthographic transparency effects evinced with frequency-matched tests. Applied Psycholinguistics, $22,571-599$.

Gleitman, L., \& Rozin, P. (1977). The structure and acquisition of reading. Relations between orthographies and the structure of language. In A. S. Rieber \& D. S. Scarborough (Eds.), Toward a psychology of reading. Hillsdale, NJ: Erlbaum.

Gombert, J. (1992). Metalinguistic development. New York: Wheatsheaf.

Goswami, U., \& Ziegler, J. C. (2006). Fluency, phonology and morphology: A response to the commentaries on becoming literate in different languages. Developmental Science, 9, 451453.

Goswami, U., Ziegler, J. C., Dalton, L., \& Schneider, W. (2003). Nonword reading across orthographies: How flexible is the choice of reading units? Applied Psycholinguistics, 24, 235-247.

Hambleton, R. K. (2005). Issues, designs, and technical guidelines for adapting tests into multiple languages and cultures. In R. K. Hambleton, P. F. Merenda, \& C. D. Spielberger (Eds.), Adapting educational and psychological tests for cross-cultural assessment. Mahwah, NJ: Erlbaum. 
Asfaha et al.: Grain size in script and teaching

Lee, S. Y., Uttal, D. H., \& Chen, C. (1995). Writing systems and acquisition of reading in American, Chinese, and Japanese first-graders. In I. Taylor \& D. R. Olson (Eds.), Scripts and literacy: Reading and learning to read alphabets, syllabaries and characters. Dordrecht: Kluwer Academic.

Liberman, I. Y., Shankweiler, D., Fischer, F. W., \& Carter, B. (1974). Explicit syllable and phoneme segmentation in the young child. Journal of Experimental Child Psychology, 18, 201-212.

Morais, J., Cary, L., Alegria, J., \& Bertelson, P. (1979). Does awareness of speech as a sequence of phones arise spontaneously? Cognition, 7, 323-331.

Nag, S. (2007). Early reading in Kannada: The pace of acquisition of orthographic knowledge and phonemic awareness. Journal of Research in Reading, 30, 7-22.

Pollard-Durodola, S. D., Cedillo, G. D., \& Denton, C. A. (2004). Linguistic units and instructional strategies that facilitate word recognition for Latino kindergarteners learning to read in Spanish. Bilingual Research Journal, 28, 319-354.

Raz, S. (1983). Tigre grammar and texts. Malibu, CA: Undena Publications.

Seymour, P. H. K., Aro, M., \& Erskine, J. M. (2003). Foundation literacy acquisition in European orthographies. British Journal of Psychology, 94, 143-174.

Share, D. (1995). Phonological recoding and self-teaching: sine qua non of reading acquisition. Cognition, 55, 151-218.

Walter, S. L., \& Davis, P. M. (2005). The Eritrea National Reading Survey. Dallas, TX: SIL International.

Winskel, H., \& Widjaja, V. (2007). Phonological awareness, letter knowledge, and literacy development in Indonesian beginner readers and spellers. Applied Psycholinguistics, 28, 23-45.

Wright, M. W. (2001). More than chanting: Multilingual literacies, ideologies and teaching methodologies in rural Eritrea. In B. Street (Ed.), Literacy and development: Ethnographic perspectives. London: Routledge.

Wydell, T. N., \& Kondo, T. (2003). Phonological deficit and the reliance on orthographic approximation for reading: A follow-up study on an English-Japanese bilingual with monolingual dyslexia. Journal of Research in Reading, 26, 33-48.

Ziegler, J. C., \& Goswami, U. (2005). Reading acquisition, developmental dyslexia, and skilled reading across languages: A psycholinguistic grain size theory. Psychological Bulletin, 131, 3-29.

Ziegler, J. C., \& Goswami, U. (2006). Becoming literate in different languages: Similar problems, different solutions. Developmental Science, 9, 429-436. 